\title{
Genetic Instability in Aging Yeast: A Metastable Hyperrecombinational State
}

\author{
M.A. MCMurRaY AND D.E. GotTsChLING \\ Division of Basic Sciences, Fred Hutchinson Cancer Research Center, Seattle, Washington 98109
}

We are all aware of changes that occur as a person reaches middle age and beyond-wrinkled skin, gray hair, poor vision, etc. But one particularly intriguing phenomenon is the dramatic rise in incidence of cancer with increasing age: About $75 \%$ of all cancers are diagnosed after the age of 55 (ACS 2004). Because cancer is typically considered a genetic disease-genetic alterations are a hallmark of tumors and the inactivation of tumor suppressor genes and/or activation of oncogenes facilitate oncogenesis (for review, see Hanahan and Weinberg 2000)_-it has been suggested that the link between aging and increased incidence of cancer may simply be a steady accumulation of genetic changes over the course of a person's life. The increased chance of attaining a sufficient number of changes to elicit oncogenesis may explain the exponential increase in cancer incidence with advancing age (Armitage and Doll 1954, 1957; Frank 2004).

However, this idea is likely too simple. For instance, the rates of spontaneous mutation observed in human tissue culture cells cannot account for the amount of genetic change observed in most tumors (Loeb et al. 2003). Furthermore, a number of nongenetic changes occur as a cell ages that could have an impact on oncogenesis. DNA methylation at gene promoters, which could inactivate tumor suppressor genes (Jones and Laird 1999), increases with age (DePinho 2000). Similarly, changes in histone modification levels, which also can impact gene expression, are observed with increasing age (DePinho 2000; Bandyopadhyay and Medrano 2003). Oxidative damage to DNA accumulates with age (Bohr 2002) and may contribute to the acquisition of mutations during carcinogenesis (Jackson and Loeb 2001).

Even if we consider only how genetic alterations contribute to the age-related increase in cancer, we can appreciate the enormous complexity of this issue. For instance, do mutations arise at a constant rate throughout an individual's life, or do mutation rates increase with age? Many biological and clinical issues confound this determination, including clonal expansion, by which an early mutation provides a growth advantage and increases opportunities for subsequent mutations; tissue-specific effects, in terms of both the frequency of tumor detection and the inherent susceptibility of certain cell types to tumorigenesis; environmental challenges, including extrinsic (e.g., carcinogens) and intrinsic (e.g., hormones) factors that arise over a lifetime and can affect tumor formation; and the continual improvement of diagnostic methods.

The budding yeast Saccharomyces cerevisiae has been developed as a model system for studying many aspects of cellular aging. Because of the asymmetric nature of its cell divisions, it has been particularly useful to study replicative life span. Replicative aging in yeast is defined by the number of mitotic cell divisions a yeast mother cell undergoes before she ceases dividing and ultimately lyses (for review, see Bitterman et al. 2003). In typical laboratory strains of haploid yeast, wild-type life spans average 20-30 divisions, during which characteristic changes affect the aging mother cell, such as increased cell size, wrinkled cell surface, and decreased division rates.

The daughters of very old mothers display all of the cellular phenotypes of old cells mentioned above, as well as a reduced life span (Egilmez and Jazwinski 1989; Kennedy et al. 1994), consistent with the daughter cells inheriting age-induced alterations. However, these traits are not stably inherited; the great-granddaughters of old mothers appear rejuvenated, with full life spans and a normal cell size and division rate (Egilmez and Jazwinski 1989; Kennedy et al. 1994). Thus, the appearance of old cell phenotypes in the daughters of old mothers is not the result of a permanent genetic change.

Nevertheless, the first proposed cause of yeast aging was centered on a genetic event: the formation of extrachromosomal rDNA circles (ERCs), which accumulate to high levels in old mother cells and are inherited by the daughters of old mothers (Sinclair and Guarente 1997). It was put forth that the ERC phenomenon represents a role for "genetic instability" in yeast aging (Jazwinski 2001; Bitterman et al. 2003). However, the actual chromosomal events creating ERCs are likely age independent. A "stochastic trigger" of recombination-mediated ERC formation early in a cell's life span, followed by exponential accumulation to a lethal threshold, can explain the sigmoidal shape of survival curves of populations of $S$. cerevisiae (Sinclair et al. 1998). Thus, the accumulation of ERCs is not a true example of age-associated genetic instability.

In an attempt to understand whether there are fundamental aspects of cellular aging that contribute to oncogenesis, we developed an assay to examine genomic instability as a function of cellular age in $S$. cerevisiae. Here we describe our surprising observations, which suggest 
that aged cells enter a metastable epigenetic state of hyperrecombination. Finally, we propose a molecular model to explain these results, with implications for the effects of aging on genomic stability in higher organisms.

\section{GENETIC INSTABILITY AND YEAST AGING}

\section{Monitoring Genome Stability throughout a Life Span: Yeast Pedigree Analysis}

In setting up a system for examining genomic instability, we chose to follow loss of heterozygosity (LOH) events, an important mechanism of tumor suppressor gene inactivation in the development of cancer (Lengauer et al. 1998). LOH may be the result of chromosome loss, recombination, or deletion (see Fig. 1). To this end we engineered a set of diploid yeast strains with heterozygosity at several different loci. Loss of the functional allele at each locus was easily assayed by colony growth under selective conditions or a change in colony color.

These marked strains were then subjected to pedigree analysis, in which a mother cell is isolated on agar media, and each of her newborn daughter cells is successively moved to a different location and allowed to form a colony. These "daughter colonies" provide a retrospective life history of the mother cell: If a genetic change such as LOH occurs during the mother's life span, it is detected by a color change, or a lack of growth when the daughter colony is transferred to selective media. If an $\mathrm{LOH}$ event occurred in the mother cell during her life span, it is evident as LOH in every daughter colony until the end of her life (e.g., Fig. 2B, fourth line from bottom). However, if the mother remained wild type and LOH occurred in a single daughter cell, it is evident as a single daughter colony with the $\mathrm{LOH}$ phenotype and subsequent wild-type daughter colonies (e.g., Fig. 2B, third line from bottom).

In our initial pedigree analyses, strains carried marker genes at various heterozygous loci, together representing six different marked chromosomes (II, III, IV, V, XII, and $\mathrm{XV})$. Among these, $\mathrm{LOH}$ at the MET15 locus on chromosome XII was observed most often $-3.2 \times 10^{-3}$ per division of a mother cell. This value contrasted with the rate of spontaneous LOH at MET15 in young (growing in culture) cells, which was significantly lower, $\sim 7 \times 10^{-4}$ per cell division, in the same strain. Thus, we found evidence of increased genetic instability in the cell divisions of aging mother cells.

More significantly though, the MET15 LOH events tended to occur in cell divisions nearer the end of life span rather than the beginning. Among the pedigrees of 16 independent mother cells with such LOH events, there were $31 \pm 3.3$ (median \pm S.E.M.) divisions before the first $\mathrm{LOH}$ event was observed, compared to $11 \pm 1.4$ divisions after the LOH event and until the end of the life span $(P<$ 0.0001 , one-tailed unpaired $t$-test). Thus, replicative aging in yeast is associated with an increased rate of MET15 LOH events in the cell divisions of old mothers.

\section{Inheriting Instability: LOH in the Progeny of Old Mothers}

In addition to $\mathrm{LOH}$ events affecting the mother or her buds, we observed an unexpected increase in the frequency of daughter colonies containing sectors of cells
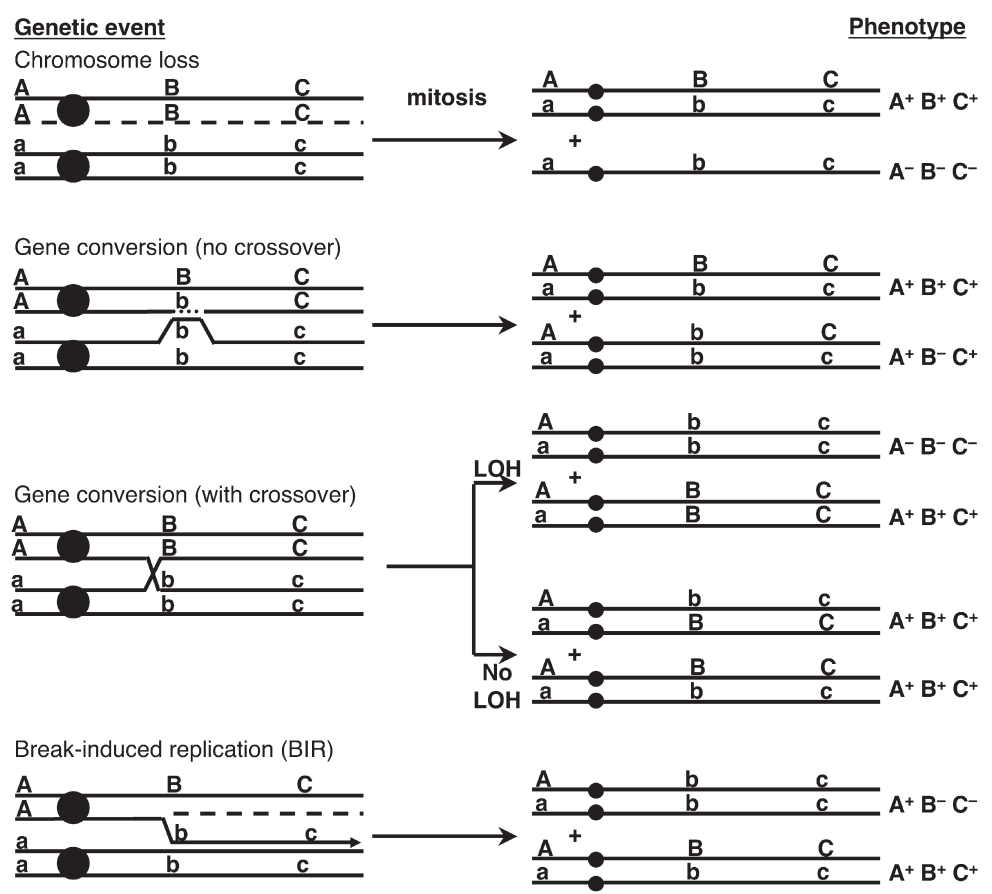

Figure 1. Mechanisms of loss of heterozygosity. For the indicated genetic event, the arrangement of alleles in each cell after mitotis (separated by "+") is given for a generic chromosome, with double-stranded DNA represented by lines, centromeres by circles, and dominant (uppercase) or recessive (lowercase) alleles at each of three loci. To the right, the phenotypes of each cell after mitosis. 
A

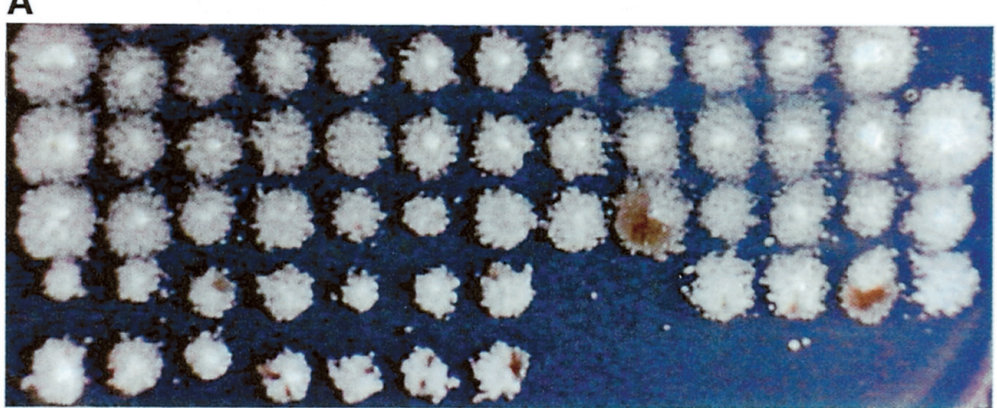

B

$\circ$, 0

$\circ$ o

O 10000000

O 0000000000

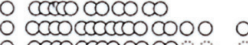

Oc000000000

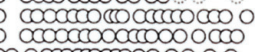

000000000000000000

- 000000 ceno 0

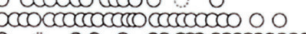

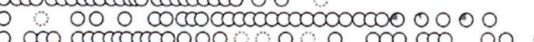

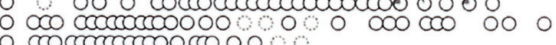

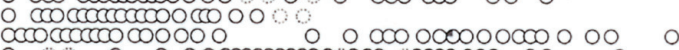

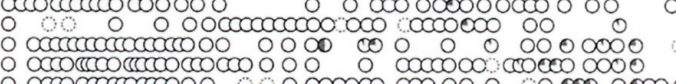

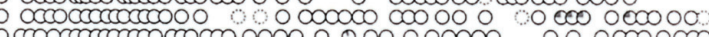

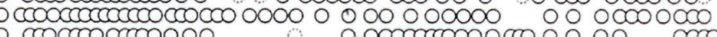

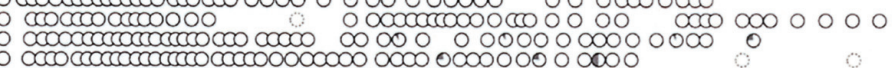

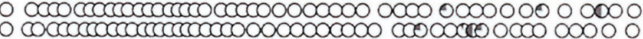

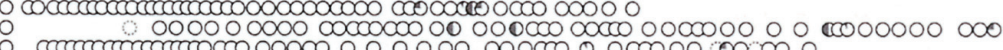

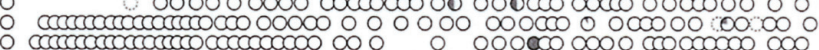

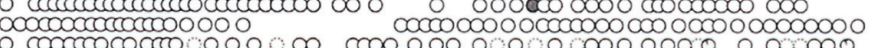

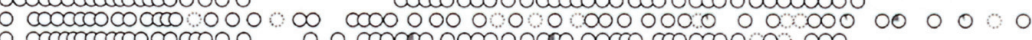

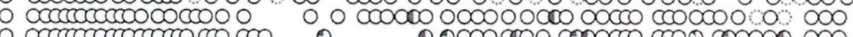

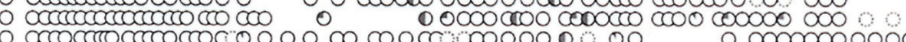

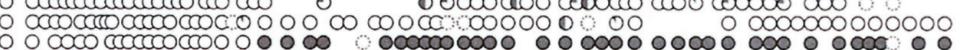

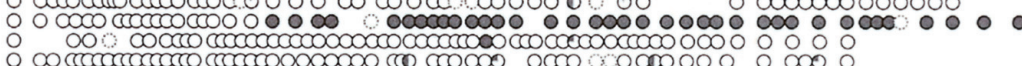

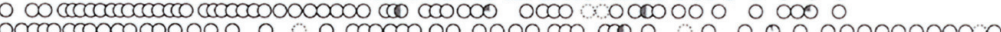

000000000000000000000000000000000000000000000000000000000000

0

2

3

4

Time (days)
C

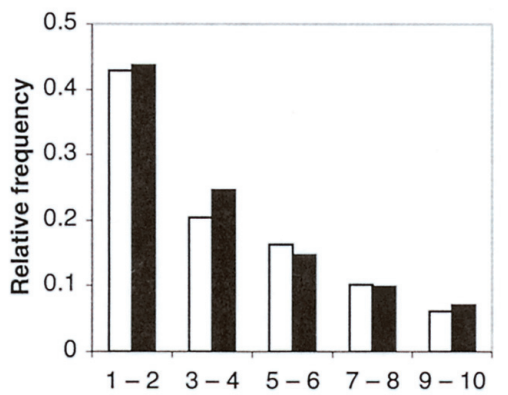

Figure 2. Pedigree analysis reveals an age-induced switch to a state of frequent LOH. $(A)$ The pedigree of a single $M E T 15 / m e t 15 \Delta$ $A D E 2 / a d e 2 \Delta$ mother cell. Colonies were formed from the individual daughter cells of a single mother cell throughout her life span of 57 cell divisions and replica plated to lead nitrate-containing media, on which met $15 \Delta /$ met $15 \Delta$ cells — resulting from MET15 LOHturn brown (Cost and Boeke 1996). The age of the mother when each daughter was produced increases left to right, top to bottom. (B) Thirty seven mother cells of the strain shown in $A$ were analyzed by pedigree analysis. Each daughter colony from a single mother is

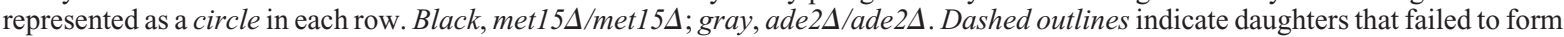
visible colonies. $(C)$ The data represented in $B$ were analyzed by counting the number of cell divisions between daughter colonies containing MET15 LOH events, defined as an eighth sector or larger of met $15 \Delta /$ met $15 \Delta$ cells. White bars show a frequency distribution of these intervals, in bins of two cell divisions. Black bars represent the expected (binomial) distribution if the probability per cell division of generating a daughter colony with a MET15 LOH event is 0.25 .

with LOH: Part of the colony remained wild type, but a significant fraction showed the phenotype associated with marker loss (Fig. 2A) (McMurray and Gottschling 2003). These events were readily detectable by exploiting the brown colony color phenotype of met 15 cells on lead nitrate media (Cost and Boeke 1996), and the red colony color phenotype of ade 2 cells on media with low adenine content (Roman 1957). Colored sectors within colonies indicate $\mathrm{LOH}$ events that occurred after the daughter cell was removed from the mother.

In the pedigree analysis, sectored daughter colonies representing $\mathrm{LOH}$ at each of the marked chromosomes were observed. These sectored colonies were most frequent when the daughter came from older mother cells. In addition, they were detected far more commonly than
$\mathrm{LOH}$ events in the aging mothers themselves (Fig. 2B) (McMurray and Gottschling 2003; data not shown). This surprising observation indicates that old mother cells produce progeny that are prone to $\mathrm{LOH}$, raising the possibility that the chromosomal instability manifested as increased LOH in the mother's divisions might be inherited by her progeny.

\section{Countdown to Instability: A Two-State Switch Running on Its Own Aging Clock}

Mother cells of the strain shown in Figure 2 divided on average 43 times (median value, $n=37$ ). (Note that these diploid cells are substantially longer-lived than other reported wild-type laboratory strains, most of which are 
haploid [Kaeberlein et al. 2004].) The first LOH eventscored as a wholly brown or brown/white daughter colony with an eighth or larger brown sector-was observed in a mother's life span after 25 divisions without an event (median value, $n=22$ ). Thereafter, $\mathrm{LOH}$ events were much more frequent, occurring on average in every third daughter colony ( $n=49$; see Fig. 2). Interestingly, this frequency did not increase as mother cells aged further (Spearman correlation coefficient $r \geq 0$ ), meaning that the rate of LOH did not continue to increase as the end of the life span approached. Furthermore, after the first LOH event in each mother's life span, the distribution of cell divisions between subsequent $\mathrm{LOH}$ events for all the mothers appeared to fit a binomial distribution, in which each cell division by the mother is associated with a constant probability $(\sim 0.25)$ of observing a daughter colony with LOH (Fig. 2C). Thus, if we consider the first appearance of an $\mathrm{LOH}$ event in a mother's pedigree to represent the onset of age-induced chromosomal instability, then the subsequent rate of $\mathrm{LOH}$ events is constant throughout the remainder of the life span. Taken together, the data suggest that after approximately 25 cell divisions by an aging mother, there is a switch-like increase in the rate of $\mathrm{LOH}$, manifested as a high frequency of $\mathrm{LOH}$ events in daughter colonies.

Among individual mother cells of the same strain, there is considerable variation in life span (Fig. 2B) (Mortimer and Johnston 1959). If the onset of increased LOH is related to the process that brings about the end of the life span, then short-lived mothers should display an earlier age of onset, whereas the onset of $\mathrm{LOH}$ should be delayed in long-lived mothers. Instead, we noticed that the onset of MET15 LOH occurred after approximately 25 cell divisions regardless of the life span of the mother (see Fig. 2B). This is perhaps best illustrated by the fact that the mothers whose pedigrees were devoid of $\mathrm{LOH}$ were significantly shorter-lived (mean life span $22 \pm 4.2$ ) than mothers with pedigrees including at least one daughter colony with $\mathrm{LOH}$ (mean life span $44 \pm 2.7$; one-tailed unpaired $t$-test $P<0.0001$ ). Thus, if a mother cell failed to achieve 25 cell divisions before dying, she was unlikely to have experienced the switch to high LOH. Furthermore, the onset of age-induced MET15 LOH was equivalent (median 23 divisions) in other $\mathrm{LOH}$ detection strains with significantly shorter life spans (median $~ 30$ ) (McMurray and Gottschling 2003; and data not shown).

We next wanted to examine more closely whether the switch to higher levels of $\mathrm{LOH}$ is causally related to the number of cell divisions from the beginning of the life span. If so, then manipulations that alter life span would not alter the age of onset of age-induced LOH. As expected, deletion of both copies of $F O B 1$, whose gene product causes the intrachromosomal rDNA recombination events that create ERCs (Defossez et al. 1999), increased the life span of diploid mother cells by $80 \%$ compared to wild type (McMurray and Gottschling 2003). However, the kinetics of the MET15 LOH events were unchanged. The median number of cell divisions in FOB 1-deleted mother cells before a first $\mathrm{LOH}$ event was 25 cell divisions, just as it was in shorter-lived wild-type cells (McMurray and Gottschling 2003). Also unchanged was the increased frequency with which subsequent $\mathrm{LOH}$ events occurred (McMurray and Gottschling 2003). Thus, prolonging life span by reducing the level of ERCs did not delay the onset nor decrease the frequency of ageinduced genomic instability. Furthermore, these data suggest that the onset of age-induced $\mathrm{LOH}$ operates on a different "clock" than does yeast replicative life span.

\section{THE MECHANISM OF LOH IN OLD CELLS}

\section{Break-induced Replication Is Responsible for Most Age-induced LOH Events}

We next determined by which pathway LOH occurred in aging yeast cells. In considering the possibilities (see Fig. 1), we found that chromosome loss was not responsible for age-induced $\mathrm{LOH}$, because single-copy markers located on the opposite arm of chromosomes with an LOH event remained intact (McMurray and Gottschling 2003). Thus, age-induced LOH is likely initiated by chromosomal damage and is not the result of chromosome nondisjunction.

It appears that age-induced $\mathrm{LOH}$ proceeds via repair of random genome-wide damage: We found that markers located further from a centromere underwent $\mathrm{LOH}$ more frequently (Table 1) (McMurray and Gottschling 2003). The greater the distance between a marker and its centromere, the higher the probability that the intervening DNA will be damaged. $\mathrm{LOH}$ can result from either reciprocal or nonreciprocal repair pathways. In reciprocal recombination (crossing over), heterozygous cells produce a mother cell homozygous for one allele, and a daughter cell homozygous for the other allele (Fig. 1). In nonreciprocal events, either the mother or the daughter loses heterozygosity, while the other remains heterozygous (Fig. 1). We found in young cells spontaneous LOH at the MET15 locus occurred primarily via crossing over (McMurray and Gottschling 2003). In contrast, the age-induced LOH events occurred predominantly by a nonreciprocal pathway (McMurray and Gottschling 2003). These results identify a mechanistic difference between the pathway of mitotic recombination normally responsible for $\mathrm{LOH}$ in young cells and the pathway resulting in increased levels of LOH as cells age.

These results suggested that age-induced $\mathrm{LOH}$ events may arise through break-induced replication (BIR) or terminal chromosome deletions. To further test this notion and distinguish between these two mechanisms, we examined the copy number of alleles present at five additional loci on the right arm of chromosome XII in clones in which age-induced MET15 LOH had occurred. In the vast majority of events ( $>99 \%$ ), LOH at MET15 was accompanied by $\mathrm{LOH}$ at all centromere-distal loci (McMurray and Gottschling 2003), consistent with our findings that centromere-distant loci are more susceptible to age-induced LOH (Table 1). Furthermore, in $>95 \%$ of MET15 LOH events, the loss of one allele at a locus was accompanied by duplication of the remaining allele (homozygosity) (McMurray and Gottschling 2003), eliminating terminal deletion as a major cause of age-induced $\mathrm{LOH}$. We conclude that BIR is the predominant pathway 
Table 1. The Frequency of Age-induced Loss of Heterozygosity (LOH) Correlates with the Centromere-Marker Distance

\begin{tabular}{lcc}
\hline Marked locus & $\begin{array}{l}\text { Distance from } \\
\text { centromere }(\mathrm{kb})\end{array}$ & $\begin{array}{l}\text { Number of } \\
\text { marker los } \\
\text { events detected }\end{array}$ \\
\hline MET15 & $2200^{*}$ & 167 \\
SAM2 & 1000 & 71 \\
GDH1 & 220 & 8 \\
\hline
\end{tabular}

The number of age-induced LOH events (daughter colonies with an eighth sector or larger of homozygous cells) observed by pedigree analysis of 79 mother cells is given for each locus examined, together with the centromere-marker interval, in kilobasepairs $(\mathrm{kb})$.

*The size of this interval depends upon the number of rDNA repeats, which was estimated as 150 .

through which age-induced LOH events occur. Thus, aging alters both the rate and the mechanism of $\mathrm{LOH}$ events.

\section{Mother Knows Best: The Asymmetry of Age-induced LOH}

As described above, BIR is an inherently nonreciprocal mechanism of $\mathrm{LOH}$, resulting in homozygosity of one cell after mitosis, but preserving both alleles in the other. Based on the presumed mechanics of BIR (Kraus et al. 2001), we predicted that these events would occur with equal likelihood in mother and daughter cells. Surprisingly, however, there was an 20 -fold daughter bias for age-induced $\mathrm{LOH}$, measured as the number of MET15 or SAM2 LOH events resulting in a homozygous daughter or mother cell (McMurray and Gottschling 2003). This observation, in combination with the previous analysis of LOH events in the mother cell herself, leads to a surprising conclusion. Although the advanced age of a mother cell drastically alters both the rate and the mechanism of LOH in her progeny, the mother cell herself rarely loses genetic information. Instead, the adverse effects on chromosomal stability induced by aging are primarily manifested in the progeny of old mothers.

\section{Does the DNA Damage Checkpoint Fail in Age-induced LOH?}

What leads to daughter-biased, BIR-mediated LOH in yeast cells as they age? We know that BIR is infrequently used to repair double-strand breaks (DSBs) in young wildtype cells (Malkova et al. 1996). Instead, DSBs are usually repaired by local gene conversion without crossing over (Malkova et al. 1996; for review see Paques and Haber 1999). In late $\mathrm{S}$ or $\mathrm{G}_{2}$, the sister chromatid is preferred as the donor for gene conversion (Kadyk and Hartwell 1992); only rare repair events in these phases utilize the homologous chromosome, a small fraction of which will be associated with crossing over (Fig.1). Of those $\mathrm{G}_{2}$ crossover events that do occur, only half will result in LOH through appropriate sister segregation in the subsequent mitoses (Fig. 1). Thus, broken chromosomes in yeast are normally repaired by non-BIR pathways - using homologous sequences to rejoin the chromosome fragments - that very rarely result in $\mathrm{LOH}$ of distal markers.
However, if the two fragments of a broken chromosome are physically separated from one another, the types of available DSB repair pathways become limited (Paulovich et al. 1997). This might occur if cells bypass a $\mathrm{G}_{2} / \mathrm{M}$ checkpoint, proceed through mitosis in the presence of an unrepaired chromosome break, and one cell inherits the chromosome fragment containing the centromere (the "centric" fragment) while the other inherits the centromere-less ("acentric") fragment (Fig. 3). In the following $\mathrm{G}_{1}$ or $\mathrm{S}$ phase, when neither the sister chromatid nor the other portion of the broken chromosome is available, the best template for repair is provided by the homologous chromosome (Fig. 3). BIR events initiating from the centric fragment of the broken chromosome will duplicate sequences from the homologous chromosome all the way to the telomere and result in $\mathrm{LOH}$ of all markers distal to the break. Thus, $\mathrm{a}_{2} / \mathrm{M}$ checkpoint defect could create situations favoring LOH through BIR by separating the two chromosome fragments created by a DSB, In this scenario, only one of the cells - either the daughter or the mother-would undergo LOH after mitosis, while the other remained heterozygous (Fig. 3).

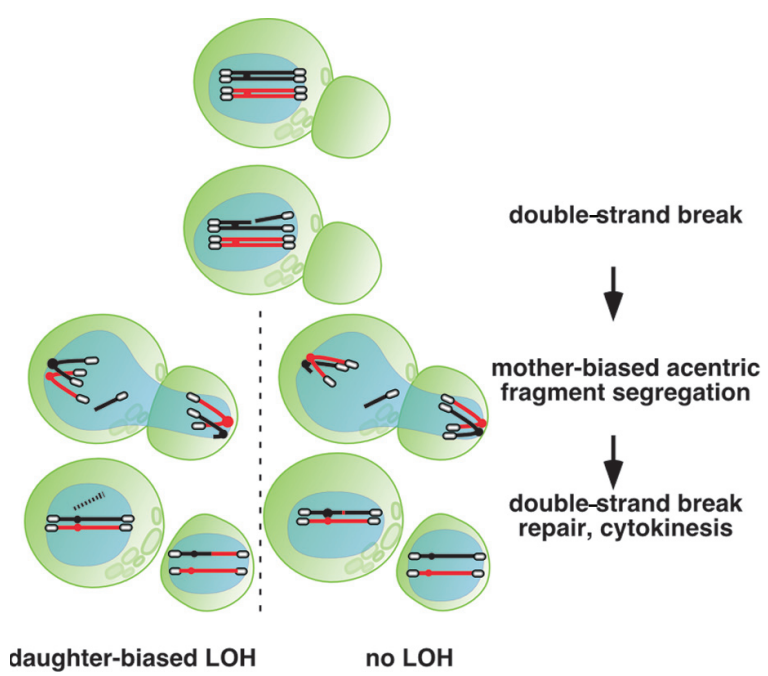

Figure 3. A model for asymmetric age-induced LOH. A diploid mother yeast cell is depicted with two homologous chromosomes (red and black; centromeres are filled circles), contained within the nucleus (blue). The cell wall is shown in green and bud scars are depicted. Top, a mother cell after DNA replication, with duplicated chromosomes. A double-strand break (DSB) in one sister chromatid of the black chromosome is followed by mitosis without repair, resulting in two potential outcomes: On the left, the broken centromere-containing chromosome fragment segregates to the daughter; on the right, it segregates to the mother. In both cases, the acentric chromosome fragment remains in the mother cell after cytokinesis. On the left, the two fragments of the broken chromosome are separated by mitosis, and repair of the broken centromere-containing fragment occurs by break-induced relication (BIR), resulting in duplication from the homologous chromosome of all sequences centromere-distal to the break. The acentric fragment remaining in the mother cell is shown to be degraded (dashed line), but could have other fates. On the right, where the mother inherits both fragments, DSB repair by nonhomologous end joining or local gene conversion without crossing over preserves both alleles at distal loci (no loss of heterozygosity [LOH]). Note that if, before DNA replication, crossing over did accompany gene conversion, LOH would not occur. 
In agreement with such a model, pronounced cell cycle arrests - while easily recognized during pedigree analysis-were essentially never detected in the cell divisions preceding the appearance of a homozygous mother or daughter cell (see Fig. 2B) (McMurray and Gottschling 2003). Thus, in the progeny of aging cells, BIR events causing $\mathrm{LOH}$ were not associated with DNA damage checkpoint-mediated cell cycle delay. Interestingly, BIR in young cells may result from $\mathrm{G}_{2} / \mathrm{M}$ checkpoint failure (Fasullo et al. 1998; Galgoczy and Toczyski 2001), offering further support for the involvement of a checkpoint defect in age-induced $\mathrm{LOH}$.

\section{Missegregation of Chromosome Fragments and Daughter-biased LOH}

How can we explain the 20 -fold daughter bias of ageinduced LOH? The answer may lie in the segregation bias of the acentric fragment of a broken chromosome. Acentric circular plasmids display a strong ( 20-fold) mother segregation bias (Murray and Szostak 1983); indeed, this is the basis of the accumulation of ERCs in aging mother cells (Sinclair and Guarente 1997). If linear chromosome fragments behave similarly to these circular molecules, then there is an inherent mother bias to the segregation of acentric chromosome fragments. As a result of random segregation of centromere-containing molecules, mother cells will often inherit both fragments of the broken chromosome after mitosis, allowing for standard mechanisms of DSB repair (Fig. 3) (Malkova et al. 1996). On the other hand, the daughter of an old mother will rarely inherit both chromosome fragments, because of the biased retention of the acentric fragment in the mother. This will result in increased rates of LOH by BIR in the following $\mathrm{G}_{1}$ of the daughter (Fig. 3). Thus, mother-biased segregation of acentric chromosome fragments may underlie the asymmetry of age-induced LOH by allowing mothers to undergo repair that conserves heterozygosity, while forcing daughters into BIR (Fig. 3).

According to our model of daughter-biased LOH, an old mother cell should often inherit the "extra" chromosome fragment that failed to segregate to the bud. What is the fate of these acentric fragments? Others have shown that BIR forks initiating from acentric molecules do not appear to replicate past the centromeres of intact chromosomes to create aneuploidy (Morrow et al. 1997). These acentric fragments may eventually be degraded in the mother during subsequent $\mathrm{S}$ phases, as previously observed for a plasmid with a single DSB (Raghuraman et al. 1994).

\section{HERITABILITY OF THE HYPERRECOMBINATIONAL STATE}

\section{Sectored Daughter Colonies Suggest Semistable Inheritance}

The detailed pedigree analysis we performed led to the discovery that old mother cells rarely experience $\mathrm{LOH}$, yet frequently produce daughter cells that do. However, the majority of age-induced LOH events were observed as sectored daughter colonies (Fig. 2B), suggesting they occurred in cell divisions after the daughter cells were removed from the mother. LOH occurring after one, two, or three generations following separation of the daughter from the mother will result in half-, quarter-, or eighthsectored daughter colonies, respectively (Fig. 4A). If the hyperrecombinational state is caused by a genetic eventsuch as mutation of a gene normally required for genome stability - it should be inherited equally by all the progeny of an old mother cell, affecting a great-great-great-granddaughter to the same extent as the mother cell herself.

By examining the frequency of half-, quarter-, and eighth-sectored daughter colonies, we asked whether a constant rate of genome instability is maintained through multiple cell divisions. Specifically, the classes of sectored colonies should be distributed predictably if the rate of $\mathrm{LOH}$ is equal for every cell. For example, the frequency of quarter-sectored colonies should be twice that of halfsectored colonies, because there are twice as many opportunities for a quarter-sectored colony to arise (Fig. 4A,B). Similarly, the frequency of eighth-sectored colonies should be four times that of half-sectored colonies.

We found the relative numbers of each class of sectored colonies among spontaneous MET15 LOH events in young cells approximated the expected ratios (Fig. $4 \mathrm{~B})$. This trend was not observed among the daughter colonies produced by pedigree analysis of old mothers. Instead, approximately the same number of eighth-sectored and half-sectored colonies were detected (Fig. 4B). This could indicate that the rate of $\mathrm{LOH}$ in every cell decreases as the cells continue to divide. Alternatively, the rate may remain high in one or a few cells for multiple generations, while all other cells have a low rate of LOH. Given the similarity in the frequency of half- and eighthsectored colonies, it is tempting to speculate that the daughter of an old mother, but not the granddaughters or more distant progeny, maintains a high rate of $\mathrm{LOH}$ for at least three cell divisions (Fig. 4A). We interpret these data as evidence that the age-induced hyperrecombinational state is inherited in a semistable manner, and that the "switch" to hyper-LOH is eventually "reset" in the distant descendants of old mothers.

\section{Resetting the Hyperrecombinational Switch}

It is worth noting that the analysis described above assumes that all cells in the early divisions of daughter colony formation enjoy full viability and similar celldoubling times. In principle, frequent cell death or cell cycle arrests could alter the appearance of sectored daughter colonies. Because our pedigree analysis did not preclude these possibilities, we addressed the heritability of the age-induced hyperrecombinational state in another way. If the state of hyperrecombination is "diluted" through the daughter lineage, then the rate of $\mathrm{LOH}$ in young cells many generations removed from an old mother should return to the rate characteristic of cultured cells. Indeed, we found that when cells taken from the daughter colonies of "postswitch" mothers (after the first 


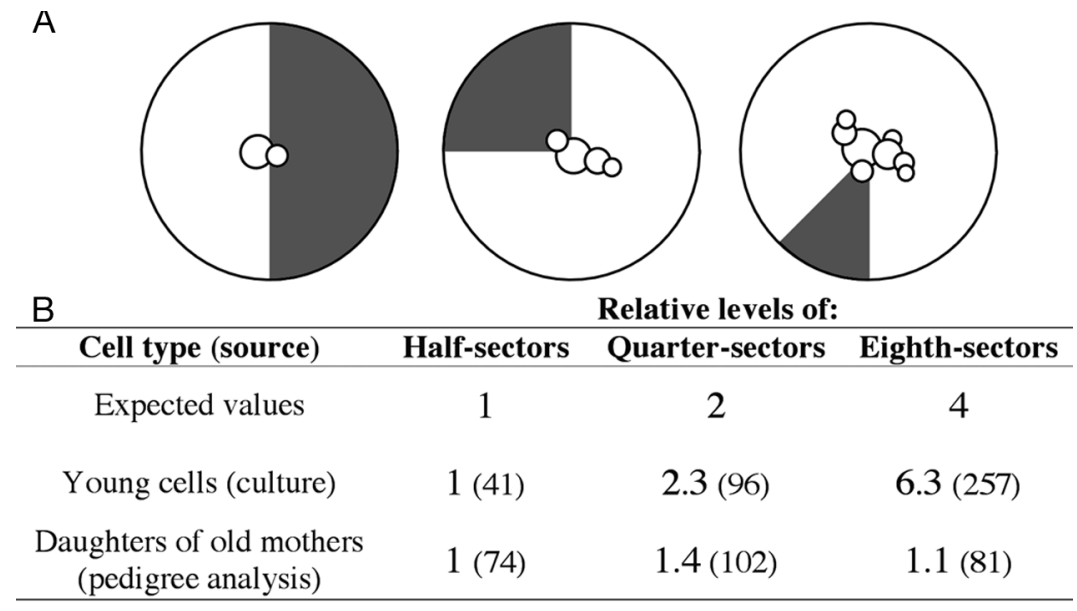

\begin{tabular}{ccc} 
C & MET15 LOH per \\
Source of cells & $\begin{array}{c}\mathbf{1 0 , 0 0 0} \text { cell } \\
\text { divisions }\end{array}$ & $\begin{array}{c}\mathbf{9 5 \%} \text { confidence interval } \\
\text { (\# half-sectored colonies) }\end{array}$ \\
\hline culture & 20 & $15-27(46)$ \\
daughter colonies & 21 & $15-28(48)$ \\
\hline
\end{tabular}

Figure 4. The age-induced hyperrecombinational state is not stably inherited. ( $A$ ) The results of loss of heterozygosity (LOH) events in various cell divisions during the growth of a colony from a single cell are depicted in the gray color within the large circles, which are superimposed upon illustrations of the relevant progenitor cell(s). Note throughout this illustration that LOH in any single cell would generate the indicated class of sectored colony; only a single possibility is shown. $(B)$ The expected proportions of each class of sectored colony are given relative to the number of half sectors, arbitrarily chosen as 1 ("Expected values"). Below, the proportions of each indicated class of sectored colony observed experimentally are presented for the indicated source of cells. Values in parentheses give the actual number of sectored colonies scored. (C) The rate of MET15 LOH was measured by the frequency of brown/white half-sectored colonies for cells of a MET15/met 15 $\Delta$ strain. As indicated, cells were either plated directly from culture or taken from the white portions of brown/white sectored daughter colonies produced by old mothers. The old mothers had previously produced multiple other daughter colonies sectored with MET15 LOH. Values represent the means of measurements from at least three independent cultures/colonies and are accompanied by the $95 \%$ confidence interval of the Poisson distribution for the number of half-sectors observed.

LOH event in the pedigree) were assayed at loci that were still heterozygous, the rate of spontaneous LOH was indistinguishable from that of cultured young cells of the same genotype (Fig. 4C; and data not shown). Because a daughter colony is composed of approximately $10^{7}$ cells, the majority of the cells assayed were approximately 25 generations removed from the original old mother. Taken together, these results suggest that the hyperrecombinational state acquired by an old mother cell and transmitted to her immediate progeny is eventually lost through the daughter lineage after many successive asymmetric cell divisions.

\section{A "SENESCENCE FACTOR" FOR GENOME STABILITY?}

The observations presented above describe a new phenotype associated with aged mother cells: chromosomal instability leading to daughter-biased loss of heterozygosity. This trait appears to be heritable, but only transiently, as it is eventually lost in the distant descendents of old cells. A similar "dilution process" affects the factor(s) causing the end of a yeast cell's life span, manifested in the gradual reestablishment of full replicative capacity in the great-great-granddaughters of old mothers (Kennedy et al. 1994). As described earlier, additional aging phenotypes are also transiently inherited by the progeny of old mothers. To explain these phenomena, others have invoked a "senescence factor," which accumulates in aging mother cells but does not affect their offspring until a critical concentration is reached, at which point the daughters of old mothers inherit senescence factor and display aging phenotypes (Egilmez and Jazwinski 1989; Kennedy et al. 1994). Accumulation with age depends on mother-biased inheritance of the factor at each cell division (Fig. 5); this asymmetry breaks down in old cells. Upon their discovery as a cause of aging, ERCs were proposed to be the senescence factor (Sinclair and Guarente 1997), and their behavior is indeed consistent with some of the aging phenomenology. However, we showed that when the formation of ERCs is suppressed, the kinetics and magnitude of age-induced LOH is unaffected (McMurray and Gottschling 2003); ERCs are not responsible for age-induced $\mathrm{LOH}$.

What could be the genome stability senescence factor? A class of candidates emerges from the recent discovery that oxidatively damaged proteins are preferentially segregated to a yeast mother cell at cytokinesis (Aguilaniu et al. 2003). Because of this asymmetric segregation, aging mother cells accumulate high levels of oxidized proteins, whereas levels in young cells are relatively low (Aguilaniu et al. 2003; Reverter-Branchat et al. 2004). However, 


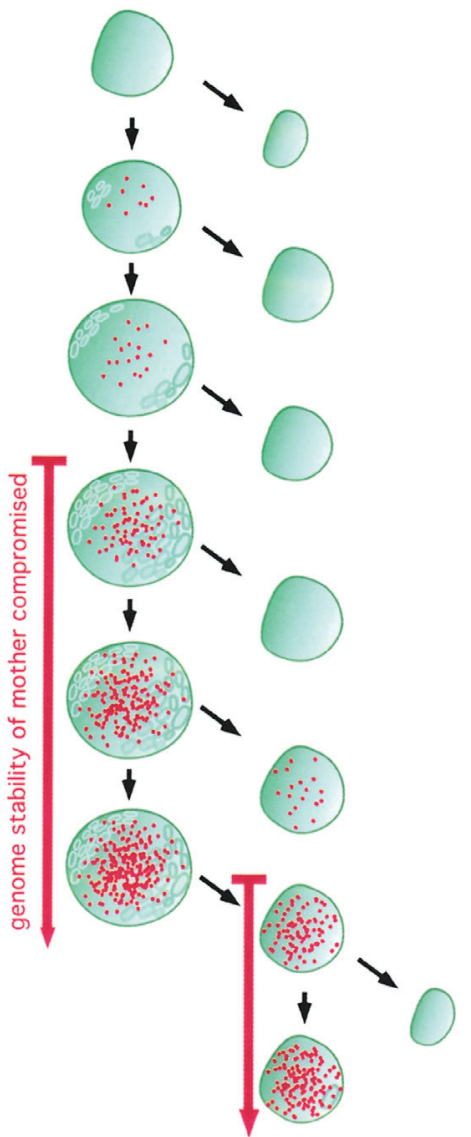

Figure 5. Model for the accumulation and inheritance of a senescence factor that affects genome stability as yeast age. On the left, a mother yeast cell undergoes replicative aging, producing a virgin daughter cell at each division, shown on the right of the mother cell. A hypothetical senescence factor (red dots) accumulates in the mother at each division, reaching high concentrations after many successive divisions. Past a certain threshold (red arrows), the concentration of the senescence factor begins to negatively impact the genome stability of the cell; the daughter of an old mother may be affected and begins accumulating more senescence factor once it becomes a mother. However, the granddaughter of an old cell (far right) does not inherit the senescence factor and retains normal genome integrity.

after the mother reaches a certain age, damaged proteins are equally distributed between her and her subsequent daughters (Aguilaniu et al. 2003). The behavior of oxidatively damaged proteins in aging cells thus fulfills the phenomenological requirements for a senescence factor.

We suggest that damaged versions of proteins critical for maintaining chromosome integrity accumulate in old cells. Once enough of the protein is in a damaged form, it acts as a "dominant negative" (Herskowitz 1987) and interferes with normal function, initiating an increased frequency of $\mathrm{LOH}$ (Fig. 5). As damaged protein continues to accumulate with increasing age of the mother cell, the damaged protein spills over into the daughter cell, and she too begins to display increased LOH. However, the amount of damaged protein is diluted away in further progeny (granddaughters, great-granddaughters, etc.), because the damaged protein is preferentially maintained in mother cells (for reasons that are unknown; Aguilaniu et al. 2003) and is replaced by new protein synthesis in daughter cells.

\section{CONCLUSIONS}

We have shown that aging yeast cells - a prospective model for human stem cells - switch to a state characterized by high rates of loss of heterozygosity. The switch does not occur stochastically during the life span, instead affecting mother cells only after approximately 25 successive cell divisions. Thus, age itself influences the rate of new genetic events. Furthermore, this apparently genome-wide effect on chromosome integrity is not itself the result of a primary genetic change: The hyperrecombinational state is inherited by the progeny of an old mother, but is eventually lost in successive cell divisions through the daughter cell lineage.

We believe these findings may be relevant as a model of human cancer. For instance, in order to explain the number of mutations, rearrangements, and other genetic events in cancer cells, a "mutator phenotype" has been postulated to represent an early alteration in tumor progression that facilitates the subsequent changes required for tumorigenesis (Loeb 1991; Loeb et al. 2003). This theory holds that, in the absence of mutator cells, a human lifetime is too short to allow the accumulation of the number of genetic events observed in cancer cells, given the low rate of events per cell division in normal cells (Loeb 1991; Loeb et al. 2003). We suggest that an epigenetic mechanism akin to what we have observed in aging yeast mother cells could provide the increase in genomic instability that is ultimately manifested in tumors, particularly in those that might originate in the stem cell compartments of older individuals.

Increased genomic instability is generally considered to be detrimental to cell vitality. If this is so, why does it occur in aging yeast cells? On one hand, this may be explained by a variation of the classical argument that natural selection cannot act upon traits manifested past the age of reproduction (Medawar 1952). For the unicellular organism $S$. cerevisiae, an old mother cell (age > 25) makes an infinitesimal $\left(1 / 2^{26}\right)$ contribution to the fitness of a clone. Thus, decreased fitness in old mothers and their progeny due to age-induced $\mathrm{LOH}$ would likely be beyond the reach of natural selection. On the other hand, age-induced genetic instability in microbial systems can also be viewed in a beneficial light. The rarity of progeny of old mother cells in an exponentially growing population may afford a degree of genotypic flexibility to the population without a significant detriment to fitness. Rare cells that have undergone genetic changes may have decreased fitness under normal conditions, but vastly increased fitness in a changing environment. In fact, such a scenario not only applies to the selective pressures incurred by a microbial system, but is also relevant when considering cells that ultimately escape normal cellular control and evolve to become cancer cells. 


\section{ACKNOWLEDGMENTS}

We thank Laurie Carr and Derek Lindstrom for their comments on the manuscript. This work was supported by a National Institutes of Health grant (AG23779) and an Ellison Medical Foundation Senior Scholar Award to D.E.G.

\section{REFERENCES}

ACS (American Cancer Society). 2004. Cancer facts \& figures 2004. American Cancer Society, Atlanta.

Aguilaniu H., Gustafsson L., Rigoulet M., and Nystrom T. 2003. Asymmetric inheritance of oxidatively damaged proteins during cytokinesis. Science 299: 1751.

Armitage P. and Doll R. 1954. The age distribution of cancer and a multi-stage theory of carcinogenesis. Br. J. Cancer 8: 1 . 1957. A two-stage theory of carcinogenesis in relation to the age distribution of human cancer. Br. J. Cancer 11: 161 .

Bandyopadhyay D. and Medrano E.E. 2003. The emerging role of epigenetics in cellular and organismal aging. Exp. Gerontol. 38: 1299.

Bitterman K.J., Medvedik O., and Sinclair D.A. 2003. Longevity regulation in Saccharomyces cerevisiae: Linking metabolism, genome stability, and heterochromatin. Microbiol. Mol. Biol. Rev. 67: 376.

Bohr V.A. 2002. Repair of oxidative DNA damage in nuclear and mitochondrial DNA, and some changes with aging in mammalian cells. Free Radic. Biol. Med. 32: 804.

Cost G.J. and Boeke J.D. 1996. A useful colony colour phenotype associated with the yeast selectable/counter-selectable marker MET15. Yeast 12: 939.

Defossez P.A., Prusty R., Kaeberlein M., Lin S.J., Ferrigno P., Silver P.A., Keil R.L., and Guarente L. 1999. Elimination of replication block protein Fob1 extends the life span of yeast mother cells. Mol. Cell 3: 447

DePinho R.A. 2000. The age of cancer. Nature 408: 248.

Egilmez N.K. and Jazwinski S.M. 1989. Evidence for the involvement of a cytoplasmic factor in the aging of the yeast Saccharomyces cerevisiae. J. Bacteriol. 171: 37.

Fasullo M., Bennett T., Ahching P., and Koudelik J. 1998. The Saccharomyces cerevisiae RAD 9 checkpoint reduces the DNA damage-associated stimulation of directed translocations. Mol. Cell. Biol. 18: 1190.

Frank S.A. 2004. Age-specific acceleration of cancer. Curr. Biol. 14: 242

Galgoczy D.J. and Toczyski D.P. 2001. Checkpoint adaptation precedes spontaneous and damage-induced genomic instability in yeast. Mol. Cell. Biol. 21: 1710 .

Hanahan D. and Weinberg R.A. 2000. The hallmarks of cancer. Cell 100: 57.

Herskowitz I. 1987. Functional inactivation of genes by dominant negative mutations. Nature 329: 219.

Jackson A.L. and Loeb L.A. 2001. The contribution of endogenous sources of DNA damage to the multiple mutations in cancer. Mutat. Res. 477: 7.
Jazwinski S.M. 2001. New clues to old yeast. Mech. Ageing Dev. 122: 865 .

Jones P.A. and Laird P.W. 1999. Cancer epigenetics comes of age. Nat. Genet. 21: 163.

Kadyk L.C. and Hartwell L.H. 1992. Sister chromatids are preferred over homologs as substrates for recombinational repair in Saccharomyces cerevisiae. Genetics 132: 387.

Kaeberlein M., Kirkland K.T., Fields S., and Kennedy B.K. 2004. Sir2-independent life span extension by calorie restriction in yeast. PLoS Biol. 2: E296.

Kennedy B.K., Austriaco N.R., Jr., and Guarente L. 1994. Daughter cells of Saccharomyces cerevisiae from old mothers display a reduced life span. J. Cell Biol. 127: 1985.

Kraus E., Leung W.-Y., and Haber J.E. 2001. Break-induced replication: A review and an example in budding yeast. Proc. Natl. Acad. Sci. 98: 8255.

Lengauer C., Kinzler K.W., and Vogelstein B. 1998. Genetic instabilities in human cancers. Nature 396: 643.

Loeb L.A. 1991. Mutator phenotype may be required for multistage carcinogenesis. Cancer Res. 51: 3075.

Loeb L.A., Loeb K.R., and Anderson J.P. 2003. Multiple mutations and cancer. Proc. Natl. Acad. Sci. 100: 776.

Malkova A., Ivanov E.L., and Haber J.E. 1996. Double-strand break repair in the absence of RAD51 in yeast: A possible role for break-induced DNA replication. Proc. Natl. Acad. Sci. 93: 7131.

McMurray M.A. and Gottschling D.E. 2003. An age-induced switch to a hyper-recombinational state. Science 301: 1908.

Medawar P.B. 1952. An unsolved problem of biology (an inaugural lecture delivered at University College, London, December 6, 1951). H.K. Lewis, London.

Morrow D.M., Connelly C., and Hieter P. 1997. "Break copy" duplication: A model for chromosome fragment formation in Saccharomyces cerevisiae. Genetics 147: 371.

Mortimer R. and Johnston J. 1959. Lifespan of individual yeast cells. Nature 183: 1751.

Murray A.W. and Szostak J.W. 1983. Pedigree analysis of plasmid segregation in yeast. Cell 34: 961.

Paques F. and Haber J.E. 1999. Multiple pathways of recombination induced by double-strand breaks in Saccharomyces cerevisiae. Microbiol. Mol. Biol. Rev. 63: 349.

Paulovich A.G., Toczyski D.P., and Hartwell L.H. 1997. When checkpoints fail. Cell 88: 315 .

Raghuraman M.K., Brewer B.J., and Fangman W.L. 1994. Activation of a yeast replication origin near a double-stranded DNA break. Genes Dev. 8: 554.

Reverter-Branchat G., Cabiscol E., Tamarit J., and Ros J. 2004. Oxidative damage to specific proteins in replicative and chronological-aged Saccharomyces cerevisiae: Common targets and prevention by calorie restriction. J. Biol. Chem. 279: 31983.

Roman H. 1957. Studies of gene mutation in Saccharomyces. Cold Spring Harbor Symp. Quant. Biol. 21: 175.

Sinclair D.A. and Guarente L. 1997. Extrachromosomal rDNA circles-A cause of aging in yeast. Cell 91: 1033.

Sinclair D.A., Mills K., and Guarente L. 1998. Molecular mechanisms of yeast aging. Trends Biochem. Sci. 23: 131. 


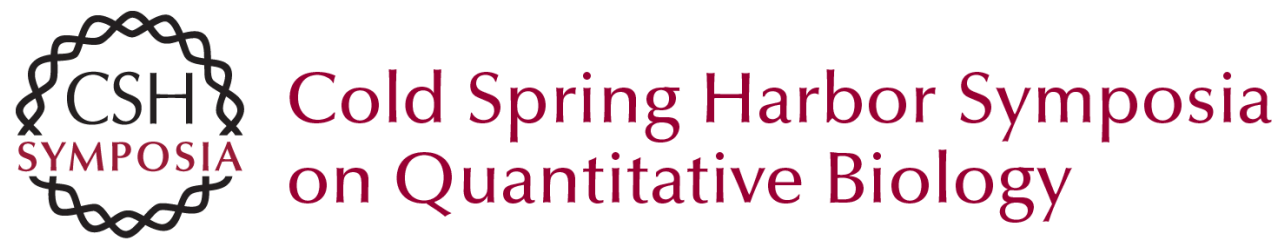

\title{
Genetic Instability in Aging Yeast: A Metastable Hyperrecombinational State
}

\author{
M.A. MCMURRAY and D.E. GOTTSCHLING \\ Cold Spring Harb Symp Quant Biol 2004 69: 339-348 \\ Access the most recent version at doi:10.1101/sqb.2004.69.339
}

References This article cites 37 articles, 16 of which can be accessed free at: http://symposium.cshlp.org/content/69/339.full.html\#ref-list-1

\section{License}

Email Alerting Receive free email alerts when new articles cite this article - sign up in Service the box at the top right corner of the article or click here. 